\title{
Prediction of Multipath Delay Profiles in Mountainous Terrain
}

\author{
Peter F. Driessen, Senior Member, IEEE
}

\begin{abstract}
Measurements of the complex impulse response of $900 \mathrm{MHz}$ radio channels in mountainous terrain in British Columbia, Canada, are used to quantify values for the normalized scattering cross section $\sigma^{0}$ for mountains covered with evergreen trees. The bistatic radar equation is then used in a propagation model to predict characteristics of the impulse response in similar terrain from topographical data.Three-dimensional (3-D) propagation models for mountainous areas are important, because such areas stress to the limit the multipath handling capabilities of most air interfaces. $\sigma^{0}$ is related to a more fundamental characteristic $\gamma$ of the surface via Lambert's law. The measured value of $\gamma$ is $\mathbf{- 2 1 . 1}$ $\pm 2.9 \mathrm{~dB}$, which is similar to some of the very few other values found in the literature. Using this value of $\gamma$, the predicted multipath delay profiles correspond well with measurements. The results can be used to predict complex impulse responses in mountainous terrain which may be convolved with a simulated data stream to predict error rate, outage or other aspects of wireless system performance.
\end{abstract}

Index Terms-Mountainous terrain, multipath delay profile, multipath prediction, propagation measurements, radio propagation, reflectance, scattering, scattering cross section, wireless communications.

\section{NOMENCLATURE}

$T \quad$ Transmitter.

$R \quad$ Receiver.

$S \quad$ Scatterer.

$\sigma^{0} \quad$ Normalized scattering cross section.

$\sigma \quad$ Scattering cross section.

$L(0) \quad$ Propagation loss of direct path.

$L\left(t_{d}\right) \quad$ Propagation loss of path delayed by $t_{d}$.

$\hat{L}\left(t_{d}\right) \quad$ Measured propagation loss of path delayed by $t_{d}$.

$h(t) \quad$ Channel impulse response.

$P_{T}(t) \quad$ Power transmitted at time $t$.

$P_{R}(t) \quad$ Power received at time $t$.

$P_{N} \quad$ Noise power.

$\rho_{m} \quad$ Signal-to-noise ratio.

$r_{T S} \quad$ Distance from transmitter $T$ to scatterer $S$.

$r_{S R} \quad$ Distance from scatterer $S$ to receiver $R$.

$r_{T R} \quad$ Distance from transmitter $T$ to receiver $R$.

$\theta_{T} \quad$ Beamwidth of antenna at $T$.

$\theta_{R} \quad$ Beamwidth of antenna at $R$.

$\theta \quad \min \theta_{T}, \theta_{R}$.

$\theta_{i} \quad$ Angle of incidence relative to surface normal.

$\phi_{i} \quad \pi / 2-\theta_{i}$.

$\theta_{r} \quad$ Angle of reflection relative to surface normal.

Manuscript received July 21, 1998; revised October 27, 1999.

The author is with the University of Victoria, Victoria V8W 3P6, BC, Canada (e-mail: driessen@uvic.ca).

Publisher Item Identifier S 0733-8716(00)01352-4.
Slope area.

$k$ th patch of area.

Area of $k$ th patch of area $S_{k}$.

Angle of incidence relative to normal of $k$ th surface element.

Angle of reflection relative to normal of $k$ th surface element.

$\begin{array}{ll}G_{R} & \text { Receive antenna gain. } \\ G_{T} & \text { Transmit antenna gain }\end{array}$

$r_{\text {cell }} \quad$ Range cell size.

$B \quad$ System bandwidth $(20 \mathrm{MHz})$.

$c \quad$ Speed of light.

$r_{\text {horiz }} \quad$ Horizontal distance of slope $A$.

$r_{\text {elev }} \quad$ Vertical distance of slope $A$.

$r_{T S}$, base $\quad$ Distance from transmitter $T$ to base of slope.

$r_{T S, \text { top }} \quad$ Distance form transmitter $T$ to top of slope.

$r_{S R}$, base $\quad$ Distance from base of slope to receiver $R$.

$r_{S R, \text { top }} \quad$ Distance from top of slope to receiver $R$.

$r_{\text {face }} \quad$ Length of slope $A$.

$r_{\theta} \quad$ Horizontal width of slope $A$.

$\theta \quad$ Beamwidth illuminating slope $A$.

$L^{0} \quad$ Additional loss due to slope angle.

$\gamma \quad$ Reflectivity of surface.

\section{INTRODUCTION}

C ELLULAR radio signals are subject to multipath propagation caused by scattering from objects in the vicinity of the transmitter and receiver. In mountainous terrain, the mountains may be visible (line-of-sight) to both transmitter and receiver and act as large reflectors. The resulting multipath signal components may be significantly delayed relative to the direct signal, and spread over a wide range of delays. The performance and reliability of digital cellular systems may be adversely affected by such delayed signal components.

The multipath propagation characteristics can be quantified by measuring the impulse response of the radio channel [1], [2]. The impulse response data (complex and time-varying) can be used to predict the performance of a digital cellular radio link. Various parameters such as the profile width, rms delay spread, and delay interval [3] can be calculated from the measured impulse response samples, and used as an indicator of potential trouble spots. The three-dimensional (3-D) propagation models for mountainous areas are important, because such areas stress to the limit the multipath handling capabilities of most air interfaces.

The need for costly measurements would be greatly reduced by using a propagation model which can predict the impulse response or some of its parameters from a topographical map 
of the radio link. Most outdoor propagation models used for coverage prediction in mountainous terrain are two-dimensional (2-D) models, i.e., they consider only the direct (shortest) path between transmitter and receiver (thus assuming that the impulse response is a delta function), and predict the attenuation (loss) on this path. Other environments do have better models, e.g., [7]. To the author's knowledge, none of the commercial propagation models and software are 3-D models which consider indirect or delayed paths in addition to the direct path, and thus cannot predict the impulse response along with the path loss.

However, several papers, as cited below, have adopted a model using the bistatic radar equation and the concept of normalized scattering cross section $\sigma^{0}$ of the mountain slopes to predict the path losses and relative delays of indirect paths from transmitter via mountain to receiver. This model can be used to construct an estimate of the impulse response, but knowledge of the value of $\sigma^{0}$ is essential to obtain meaningful results.

If we assume a Lambertian scattering model for the terrain, then the normalized scattering cross section $\sigma^{0}=\gamma \cos \theta_{i}$ where $\gamma$ is a property of the surface element, and $\theta_{i}$ is the angle of incidence relative to the normal vector $n$ which defines the orientation of the surface. For the Lambertian model, $\gamma$ is a fundamental quantity or parameter which is intrinsic to the mountain surfaces, whereas $\sigma^{0}$ depends on path-slope geometry. Other models with more parameters are reviewed in Section III.

In the first part of this paper, we present the results of an extensive campaign of impulse response measurements in many sites located near the mountains in Vancouver and Victoria, Canada. The sites were selected to facilitate obtaining reliable measurements of $\sigma^{0}$, and then using the angle of incidence to derive a value of $\gamma$. The experimental values for $\gamma$ obtained from this data were remarkably consistent for different mountain slopes, all covered with evergreen trees.

In the second part, we use this value of $\gamma$ with the bistatic radar propagation model and Lambertian scattering to predict multipath delay profiles.

While the measurements are confined to $900 \mathrm{MHz}$, it is expected that the same propagation model can be applied at different frequencies, such as $1900 \mathrm{MHz}$, with only a minor change in the value of $\gamma$.

The paper is organized as follows. The propagation model is presented in Section II, and a literature review in Section III. The measurement program is described in Section IV, and values of $\sigma^{0}$ and $\gamma$ are derived from the experimental results in Section V. Predictions of multipath delay profiles from the topo map are presented in Section VI, with discussion and conclusions in Section VII.

\section{Propagation Model}

Models of the propagation loss $L_{\text {delayed }}=L\left(t_{d}\right)$ of a delayed path scattered from mountainous terrain have been presented in [4], [5], [10]-[12], [17], and [23] based on the bistatic radar equation

$$
L\left(t_{d}\right)=\frac{P_{R}\left(t=\left(r_{T S}+r_{S R}\right) / c\right)}{P_{T}(t=0)}=\frac{\lambda^{2} \sigma}{(4 \pi)^{3} r_{T S}^{2} r_{S R}^{2}}
$$

where

$$
\begin{array}{ll}
\lambda & \text { wavelength; } \\
\sigma & \text { scattering cross section of the target; } \\
P_{T}, P_{R} & \text { transmitted and received powers; } \\
r_{T S}, r_{S R}, r_{T R} & \text { transmitter/scatterer, scatterer/receiver, } \\
& \text { and transmitter/receiver path lengths. }
\end{array}
$$

Furthermore, $t_{d}=r_{d} / c$ with $r_{d}=r_{T S}+r_{S R}-r_{T R}$ is the relative delay between direct and delayed paths and $t=0$ is a time reference. If we transmit a signal with power $P_{T}(t)=$ $P_{T}(t=0) \delta(t)$, then $L\left(t_{d}\right)$ is the path loss for the path with absolute delay $t=\left(r_{T S}+r_{R S}\right) / c$. We also define $L_{\text {direct }}=$ $L\left(t_{d}=0\right)=\left(P_{R}\left(t=R_{T R} / c\right)\right) /\left(P_{T}(t=0)\right)$ as the path loss on the direct path between transmitter and receiver. $L(0)$ may be determined by existing propagation models, e.g., [6]. The overall propagation loss can be represented by the channel impulse response $|h(t)|^{2}$, where for this case of one indirect path

$$
|h(t)|^{2}=L(0) \delta\left(t-r_{T R} / c\right)+L\left(t_{d}\right) \delta\left(t-\left(r_{T S}+r_{S R}\right) / c\right) .
$$

In practice, the $\delta(t)$ are replaced by pulses with a finite time duration of the order of the channel bandwidth, $B$. It remains to evaluate $L\left(t_{d}\right)$.

If the target is a single uniform mountain slope, we may approximate $\sigma=\sigma^{0} A$ in (1), where $\sigma^{0}$ is the normalized radar cross section of the mountain slope, and $A$ is the area of the mountain slope illuminated by the transmitter and visible from the receiver. For general nonuniform mountain slopes, we define the normalized radar cross section $\sigma^{0}=d \sigma / d A$ [8], and (1) may be written

$$
L\left(t_{d}\right)=\frac{\lambda^{2}}{(4 \pi)^{3}} \int_{A} \frac{\sigma^{0} d A}{r_{T S}^{2} r_{R S}^{2}} .
$$

This integral can in principle be evaluated as a sum by defining the elements of area $d A$ and their orientation using a topographical data base of elevations and an estimate of $\sigma^{0}$ for each element $d A$. In (3), we select only those elements $d A$ in a range cell for which $r_{T S}+r_{S R}$ is constant to within the distance resolution $c / B$, so that $L\left(t_{d}\right)$ is the weight of the impulse response at time delay $t_{d}$ relative to the direct path. Thus the integral becomes the sum

$$
L\left(t_{d}\right)=\sum_{k} \frac{\lambda^{2}}{(4 \pi)^{3}} \frac{\sigma_{k}^{0} d A_{k}}{r_{T S_{k}}^{2} r_{S_{k} R}^{2}}
$$

where $\sigma_{k}^{0}$ is the normalized scattering cross section of the patch of area $S_{k}$ with area $d A_{k}$, and the patches $k$ are chosen such that $r_{T S_{k}}+r_{S_{k} R} \epsilon\left(r_{d}-c / B, r_{d}+c / B\right)$.

By performing the integral over different range cells, the squared magnitude of the channel impulse response is obtained as

$$
|h(t)|^{2}=L(0) \delta\left(t-r_{T R} / c\right)+\sum_{t_{d}} L\left(t_{d}\right) \delta\left(t-t_{d}-r_{T R} / c\right)
$$

where the $L(\cdot)$ are local mean or spatial average values. 
For a mountainous region, all $S_{k}$ are modeled as rough surface scatterers or diffuse reflectors. We consider the Lambertian scattering model, but other models such as the generalized non-Lambertian model may be used. For the Lambertian model, $\sigma_{k}^{0}=\gamma \cos \theta_{i, k}$ where $\gamma$ is a property of the surface, and $\theta_{i, k}$ is the angle of incidence relative to the normal vector $n_{k}$ which defines the orientation of $S_{k}$. For fixed $\theta_{i, k}$, the reflectance does not change as the observer moves (i.e., as $\theta_{r, k}$ the angle of reflection changes). For an alternative model [15], [17], $\sigma_{k}^{0}=\gamma \cos \theta_{i, k} \cos \theta_{r, k}$, so that the reflectance does change with the angle of reflection. A more sophisticated model [20], [21] assumes that each $S_{k}$ with area $d A_{k}$ is made up of many Lambertian facets $d a$ such that $\lambda^{2} \ll d a \ll d A$ with different orientations. In this model, the reflectance of the surface increases as the observer moves toward the source direction.

It is important to note that, from measurements of terrain scattering [23], $\sigma_{k}^{0}$ is essentially constant for $\theta_{i, k}, \theta_{r, k}$ not near normal or grazing incidence, but increases rapidly for angles near normal $\left(0^{\circ}\right)$ and decreases near grazing $\left(90^{\circ}\right)$.

For large distributed targets such as mountain slopes (in contrast with point targets such as isolated buildings), it is reasonable to assume that the differences in $\sigma^{0}$ within the area of integration are smoothed out [4]. Thus a much coarser grid of areas $d A$ which include complete major mountain slopes in one or two elements $d A$ can be used. With this approach, the range cells are of size $r_{\text {cell }} \gg c / B$ such that each term in (5) corresponds to a major mountain slope (or significant portion thereof) visible from both transmitter and receiver. The impulse response is approximated by (5), where $L\left(t_{d}\right)$ in each term is the sum of all components within these large range cells centered at $r_{T S}+r_{S R}=r_{d}+r_{T R}$, and the delta functions $\delta(t)$ are replaced by broad pulses or clusters $p(t)$ with unit area and nominal width $r_{c e l l} / c$. Thus (3) for a particular mountain slope is simplified to

$$
L\left(t_{d}\right)=\frac{\lambda^{2}}{(4 \pi)^{3}} \frac{\sigma^{0} A}{r_{T S}^{2} r_{S R}^{2}}
$$

where $A$ is the area of the mountain slope (width $\times$ height) estimated from a topographical map. The estimate of $A$ is not critical, since a $50 \%$ error in the estimate of $A$ yields only a $3-\mathrm{dB}$ error in the value of $L\left(t_{d}\right)$. The precise steepness of the slope is also not critical (provided it is not near vertical or horizontal [23]), since $\sigma^{0}$ changes only as the sine of the slope angle [23] according to the Lambertian model. The resulting error in $L\left(t_{d}\right)$ is acceptable for our purposes. By using large range cells, the number of terms in (5) is reduced to one or two for each mountain slope considered.

\section{LITERATURE REVIEW}

The concept of normalized scattering cross section $\sigma^{0}$ and reflectance $\gamma$ based on the bistatic radar equation has been used to develop models for propagation in mountainous terrain. In the earliest reference found [10], $\gamma$ is calculated as the product of a surface roughness factor (which depends on the angle of incidence, and the variance of the normal distribution for surface roughness)[8] and the reflection coefficient (which depends on the dielectric constant and conductivity of the surface). Using literature values for poor ground $(\epsilon=15, \sigma=0.001$
TABLE I

LITERATURE DATA FOR NORMALIZED SCATTERING CROSS SECTION AND REFLECTANCE

\begin{tabular}{|c|c|c|c|c|c|}
\hline $\begin{array}{l}\text { reference } \\
{[10]}\end{array}$ & $\begin{array}{l}\text { frequency }(\mathrm{MHz}) \\
432\end{array}$ & $\sigma^{0}(\mathrm{~dB})$ & $\theta_{i}$ & $\theta_{r}$ & $\gamma(\mathrm{dB})$ \\
\hline [16] & 900 & -10 & & & \\
\hline [14] & 900 & 28 & & & \\
\hline [15] & $900 / 1900$ & $-10 /-13$ & & & \\
\hline [17] & 900 & -21 & 51 & 53 & $(-16.6)$ \\
\hline [26] & 442 & -13 to -19 & & & \\
\hline [13] & 910 & -21 to -30 & & & \\
\hline [22] & $\mathbf{L}$ band & -17.5 & 55 & & $(-15.1)$ \\
\hline [22] & $\mathbf{L}$ band & -11.0 & 35 & & $(-10.1)$ \\
\hline [23] & $\mathrm{L}$ band & -16 & 30 & & $(-15.4)$ \\
\hline [24] & $\mathrm{L}$ band & -22 & 20 to 65 & -21.6 to -18.3 & \\
\hline
\end{tabular}

TABLE II

MEASUREMENT Sites, FILE NAmes

$\begin{array}{llllll}\text { location name } & \text { figures } & R_{1}, S_{1} & R_{2}, S_{1} & R_{1}, S_{2} & R_{2}, S_{2} \\ & & & & & \\ \text { Malahat } & 1,2 & \text { motela-b } & \text { malpk } & & \text { malpa } \\ \text { Cowichan Bay } & 3,4 & \text { cbaym-p } & \text { cbayd-i } & \text { cbssa-b } & \text { cbb } \\ \text { Cowichan Lake } & - & \text { cowb-f } & & & \\ \text { Vancouver } & - & \text { brock } & \text { grouse } & & \end{array}$

siemens $/ \mathrm{m}$ ), the reflection coefficient for normal incidence is $9 \mathrm{~dB}$. Since the number of elementary scatterers $d A$ is large and have random phases, the loss $L\left(t_{d}\right)$ in (3) follows a Rayleigh probability density, and the median value of the loss will be $11.6 \mathrm{~dB}$ below the maximum calculated value (analogous to the Lambertian model). Thus according to these assumptions, $\gamma \simeq-21 \mathrm{~dB}$.

Some measured values for $\sigma^{0}$ were found in the literature. In [17], $\gamma$ was measured to be $-21 \mathrm{~dB}$ for a target hill densely covered with evergreen and deciduous trees. In [16], as cited in [17], $\sigma^{0} \simeq-7$ to $-10 \mathrm{~dB}$ for steep cliffs with no trees. In [14], $\sigma^{0}=-28 \mathrm{~dB}$, whereas in [15], the same authors obtain $\sigma^{0}=-10 \mathrm{~dB}$. In [12] using data from [26] at $442 \mathrm{MHz}, \sigma^{0}$ is in the range -13 to $-19 \mathrm{~dB}$. In [13], $\sigma^{0}$ is measured in Vancouver in the -21 to $-30 \mathrm{~dB}$ range. In [22], p. 148, for vertical-to-vertical $(\mathrm{VV})$ polarization at $\mathrm{L}$ band, $\sigma^{0}=-17.5 \mathrm{~dB}$ with standard deviation $2.1 \mathrm{~dB}$ with a $\phi_{i}=55^{\circ}$ incidence angle $\left(\theta_{i}=35^{\circ}\right.$ from normal). (The result for $\mathrm{HH}$ polarization is almost the same.) In [23], $\sigma^{0} \simeq-15 \mathrm{~dB}$ for woods with $\phi_{i}=60^{\circ}$. In [24], $L$ band VV scattering from tree trunks is about $-22 \mathrm{~dB}$ in the range from $20^{\circ}$ to $65^{\circ}$. Note that in these latter three results, the trees are on a flat horizontal surface and the wave arrives at an angle to the tree trunks, whereas in our measurements, the surface is sloped, but the wave arrives perpendicular to the tree trunks. These literature values for $\sigma^{0}$ or $\gamma$ are summarized in Table I. Bracketed values are calculated using either the values of the incident angle only (Lambertian model) or both incident and reflected angle (alternate model), where these angles are given.

These results [Table I] suggest that both $\sigma^{0}$ and $\gamma$ are in the -10 to $-30 \mathrm{~dB}$ range, and the angular dependence of $\sigma^{0}$ is not clear. These results are insufficient to be used with confidence, and thus the measurement program was undertaken.

\section{EXPERIMENTAL PROGRAM}

The transmitter $T$ and receiver $R$ locations for the impulse response measurements were chosen such that both $T$ and $R$ had 
a clear line-of-sight to the mountain slope $S$. The measurement of received power is converted to a measurement of $\sigma^{0}$ via the link budget parameters, as shown below in Section V.

The $T$ antenna is an 18-element vertically polarized yagi [25] with nominal $E$ and $H$ plane beamwidth of $30^{\circ}$, and the $R$ antenna array is either a $\lambda / 4$ monopole, or an array of four of these yagis spaced horizontally by $2 \lambda$ yielding an $H$ plane beamwidth of $10^{\circ}$. To obtain a reliable measurement of $\sigma^{0}$, the mountain slopes were selected to be as flat and uniform as possible over the area illuminated by the $R$ antenna, i.e., to have a uniform slope over the horizontal distance subtended by the $10^{\circ}$ beamwidth and the vertical distance from bottom to top.

A total of six mountain slopes were found which met the flatness criteria, and where the surrounding terrain had accessible locations for both $T$ and $R$ to have a clear line of sight to $S$. For four of these slopes there were two $R$ locations available, so measurements could be made with two different values of $\theta_{r}$. Maps and descriptions are included in the next section (cf. Table II).

The propagation analyzer equipment [11] used to gather the impulse response samples consists of a $910 \mathrm{MHz} 10 \mathrm{Mb} / \mathrm{s}$ BPSK transmitter with a 1023 bit PRBS ( $1.023 \mathrm{mS}$ sequence length) and a sliding correlator receiver [9] with a $1 \mathrm{KHz}$ data rate offset. The resulting correlation time to obtain one complex impulse response samples is $1.023 \mathrm{~s}$ (time scaling of $10 \mathrm{Mb} / \mathrm{s} \div 1 \mathrm{Khz}$ $=10^{4}$ ), and the space resolution is one-half chip equivalent to $15 \mathrm{~m}$. Rubidium clocks were used at both transmitter and receiver to obtain correct $I$ and $Q$ components of $h(t)$, sampled at a $4 \mathrm{KHz}$ rate, yielding one sample every $25 \mathrm{~ns}$. Absolute delay of the channel can also be measured. Transmitter power was $+33 \mathrm{dBm}$ or $2 \mathrm{~W}$. At the receiver antenna, the noise power was $P_{N}=-98 \mathrm{dBm}$, arising from connectors and a $20 \mathrm{MHz}$ bandpass filter with 1.5-dB insertion loss, a 1.4-dB noise figure preamp with $36-\mathrm{dB}$ gain, and a cable with 9-dB loss to the receiver in the mobile van.

\section{ESTIMATE OF $\sigma^{0}$ FROM EXPERIMENTAL RESULTS}

The measured impulse response samples consist of one or more distinct clusters at different delays, which can be interpreted in terms of the topography. Each cluster was identified with the particular mountain slope for which the indirect path length $r_{T S}+r_{S R}=r_{d}+r_{T R}$ from transmitter via mountain slope to receiver corresponds with the average delay of the cluster. We wish to compare the values of $L\left(t_{d}\right)$ in the propagation model (4) and (5) with estimates $\hat{L}\left(t_{d}\right)$ obtained from the measured power in each cluster. This measured power is determined by summing the values of $P_{R}(t)$ over the range of delays of the cluster. The measured propagation loss over this delayed path is then

$$
\hat{L}\left(t_{d}\right)=\frac{1}{P_{T}(t=0)} \sum_{t=\left(r_{T S}+r_{R S}-r_{\text {cell }} / 2\right) / c}^{\left(r_{T S}+r_{R S}+r_{\mathrm{cell}} / 2\right) / c} P_{R}(t)<1 .
$$

The value of $\sigma^{0}$ for the mountain slope can be estimated by comparing $\hat{L}\left(t_{d}\right)$ with $L\left(t_{d}\right)$ in (4).

$\hat{L}\left(t_{d}\right)$ is obtained from the measured signal-to-noise ratio $\rho_{m}$ of the cluster, where, in decibels,

$$
\rho_{m}=P_{R}-P_{N}=P_{T}+G_{T}+G_{R}+\hat{L}\left(t_{d}\right)-P_{N} .
$$

The value of $\rho_{m}$ is obtained from the measured impulse response $h(t)$, as shown in the Appendix.

\section{A. Link Budget}

From the link budget (8), $\rho_{m}$ can be expressed in terms of the measured $\sigma^{0}$ and known parameters as

$$
\begin{aligned}
\rho_{m}= & P_{T}+G_{T}+G_{R}+L\left(t_{d}\right)-P_{N} \\
= & P_{T}+G_{T}+G_{R} \\
& +\left(-42.6+10 \log \sigma^{0}+10 \log \frac{A}{r_{T S}^{2} r_{S R}^{2}}\right)-P_{N}
\end{aligned}
$$

using (4) with $r_{T S}, r_{S R}, \lambda$ in meters, and $A$ in meters squared. Rearranging and substituting $P_{N}=-98$, we express the measured $\sigma^{0}$ in terms of the system, geometric and measured parameters

$$
\begin{aligned}
& 10 \log \sigma^{0}(d B) \\
& \quad=-P_{T}-G_{T}-G_{R}+42.6-10 \log \frac{A}{r_{T S}^{2} r_{S R}^{2}}-98+\rho_{m} .
\end{aligned}
$$

The reliability of the $\sigma^{0}$ estimate is increased by obtaining estimates from many different impulse response samples containing a delayed cluster arising from the same mountain slope.

\section{B. Description of Measurement Sites}

A total of six mountain slopes on four maps were found which met both the flatness and clear line-of-sight criteria (maps, delay profiles, Figs. 1-4). For any one map, there was one transmit location $T$, and one or two slopes $S_{i}$ and one or two views (receive locations) $R_{i}$ per slope. File names for each pair of $S_{i}, R_{i}$ locations is given in Table II. The slope flatness criterion, where the approximation (4) is reasonable, is met by choosing slopes with parallel contour lines on the maps. The clear line-of-sight criterion on both $T S$ and $S R$ paths is met by choosing slopes where these paths were over water. The $T$ and $R_{i}$ locations and mountain slopes $S_{i}$ are marked on the maps.

\section{Analysis details}

The values of $A, r_{T S}, r_{S R}$ in (10) as well as $\theta_{i}, \theta_{r}$ are determined from the maps as follows. We obtain the following parameters directly from the map (Fig 5): $r_{\text {horiz }}, r_{\text {elev }}, r_{T S}$, base, $r_{T S, \text { top }}, r_{S R \text {, base }}, r_{S R, \text { top }}$. We define $A=r_{\text {face }} r_{\theta}$ from Fig. 5 where $r_{\text {face }}=\sqrt{r_{\text {horiz }}^{2}+r_{\text {elev }}^{2}}$ and $r_{\theta}=r_{S R} \cdot 2 \tan (\theta / 2)$ and $\theta$ is the minimum horizontal beamwidth $\left(\theta_{T}\right.$ or $\left.\theta_{R}\right)$. The grazing angle to the face $\phi_{i}=\pi / 2-\theta_{i} \simeq \arctan \left(r_{\text {elev }} / r_{\text {horiz }}\right)$. The path lengths from $T$ and $R$ to the base and top of the slope measured from the map are used to find the distances $r_{T S}, r_{S R}$ to the center of the slope, as well as the minimum and maximum path difference $\delta r_{\min }=r_{T S, \text { base }}+r_{S R \text {, base }}-r_{T R}, \delta r_{\max }=$ $r_{T S, \text { top }}+r_{S R \text {, top }}-r_{T R}$, used to define the beginning and end of the cluster. Using the Lambertian model, the measured value of $\sigma^{0}=\gamma \cos \theta_{i}$, thus in $\mathrm{dB}$ the loss factor $L^{0}\left(\theta_{i}\right)=$ $10 \log \cos \theta_{i}=10 \log \sin \arctan \left(r_{\text {elev }} / r_{\text {horiz }}\right) \leq 0$ and in $\mathrm{dB} \gamma=\sigma^{0}-L\left(\theta_{i}\right) \geq \sigma^{0}$. 

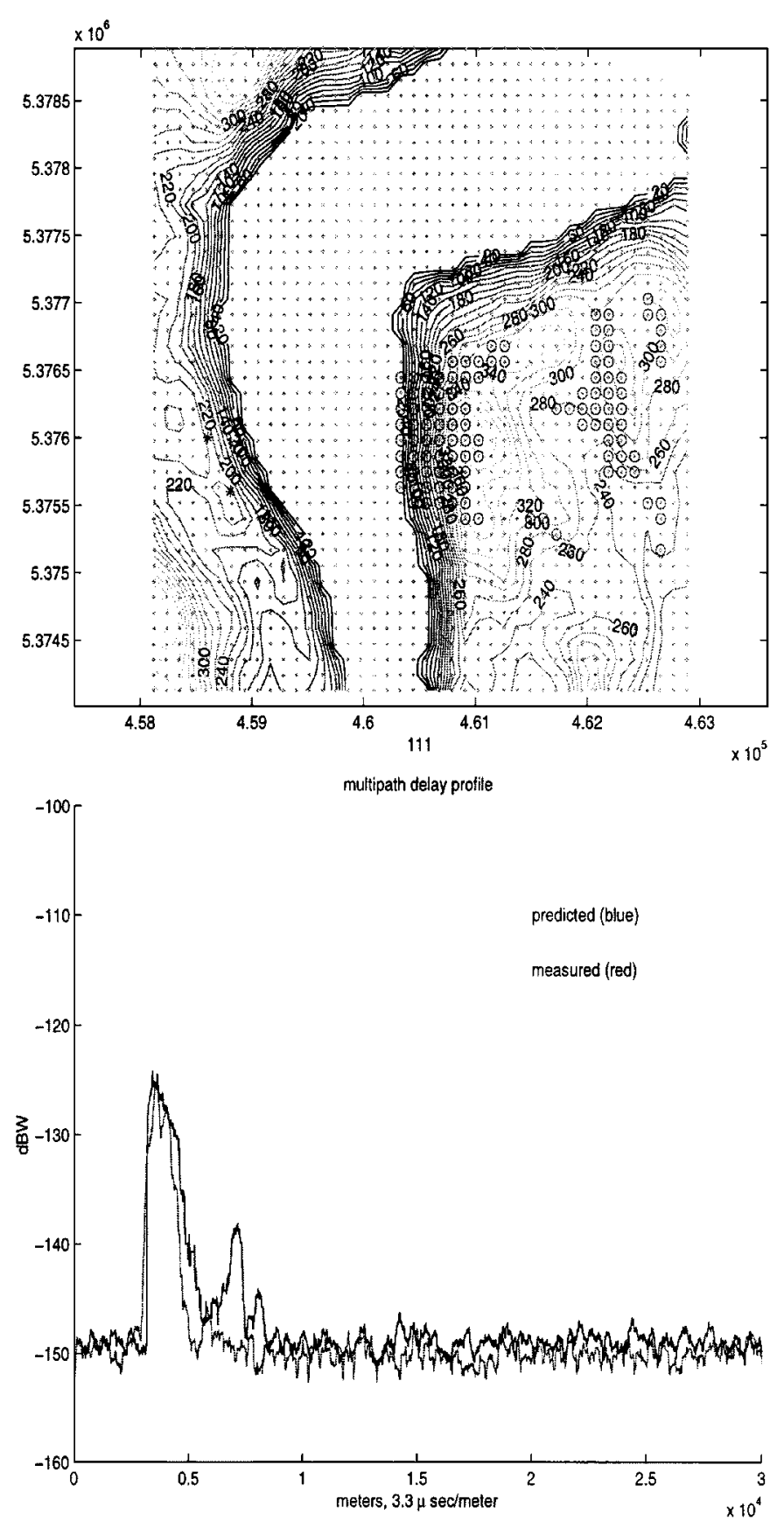

Fig. 1. Multipath delay profile—Malahat, near Victoria, BC, Canada.

\section{Measurement Results for $\sigma^{0}$}

The measured values of $\sigma^{0}$ are shown in Table III as determined from (10) for the parameters of each measurement site in Table II. The mean and standard deviations for $\sigma^{0}$ and $\gamma$ are computed from the ensemble of multipath delay profiles in the named files (e.g., motela1-50). $\gamma$ is remarkably consistent over the range of mountain slopes considered, and also consistent with many of the literature values. Maps and measured delay profiles are given in the next section.

An approximate overall estimate for $\sigma^{0}$ and $\gamma$ for the entire measurement campaign may be obtained by assuming the results from each location and angle are Gaussian, and computing the mean and standard deviation for all the data. The
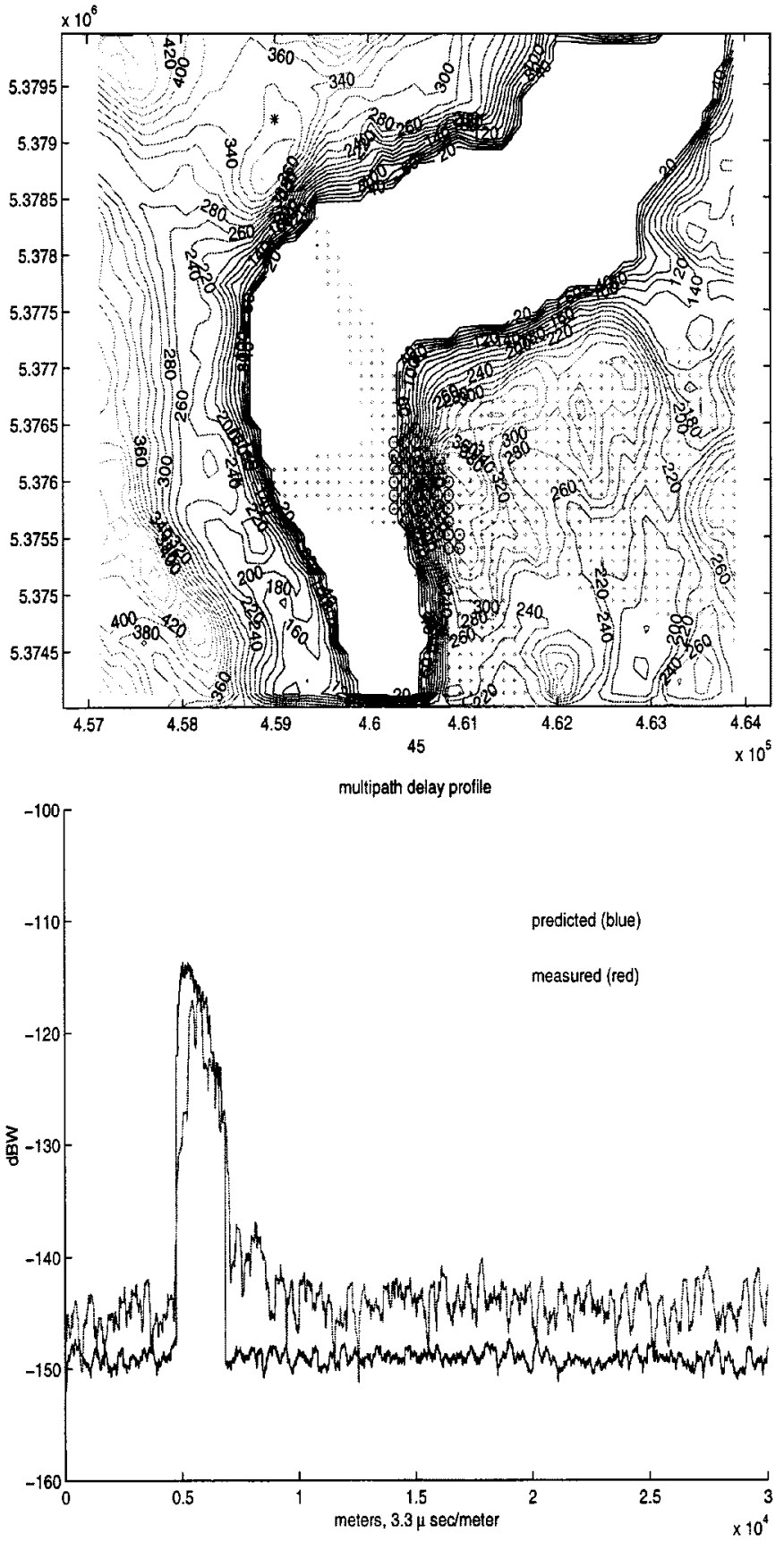

Fig. 2. Multipath delay profile-Malahat, near Victoria, BC, Canada.

mean \pm standard deviation results are $\sigma^{0}=-24.7 \pm 4.1 \mathrm{~dB}$, $\gamma=-21.1 \pm 2.9 \mathrm{~dB}$. Fig. 6 shows the corresponding histograms. Thus using the Lambertian model $\sigma^{0}=\gamma \cos \theta_{i}$ to compensate for different $\theta_{i}$ results in $\gamma$ having a significantly lower standard deviation than $\sigma^{0}$. Thus $\gamma$ is preferred over $\sigma^{0}$ as a fundamental parameter which characterizes the terrain scattering.

The results presented here provide a "missing link" in a 3-D propagation prediction method that considers multiple paths in addition to the direct path, and provides average path loss, delay profiles and rms delay spreads in mountainous environments. These environments are very important because they stress to the limit the multipath handling capabilities of most air interfaces. At the same time, there seem to be no good tools for 

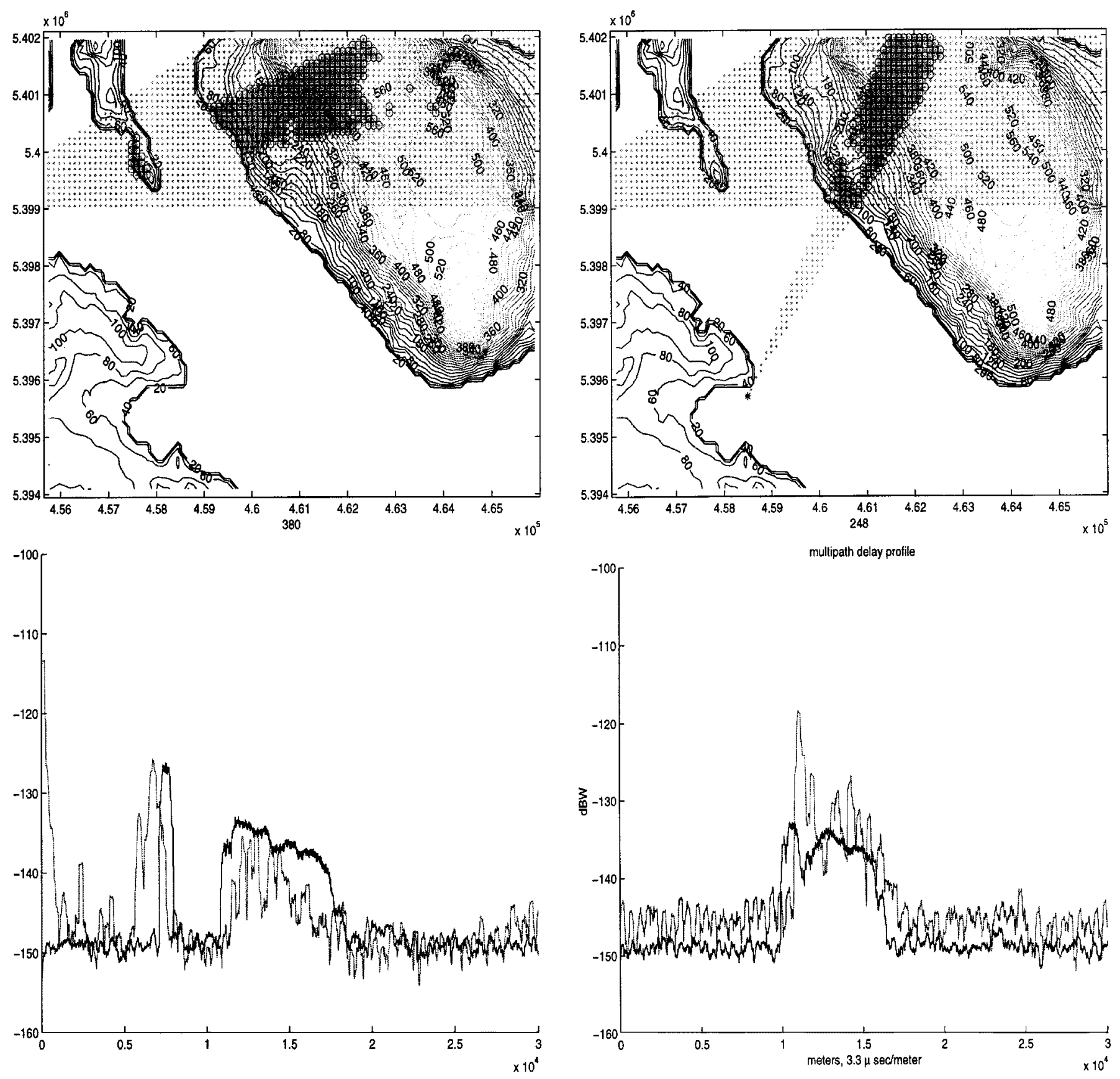

Fig. 3. Multipath delay profile—Cowichan Bay, near Victoria, BC, Canada.

propagation prediction in mountain areas, and insufficient modeling of mountain-area multipath, in contrast to other environments, e.g., [7]. The topo-map and radar scattering cross section based approach presented here is very promising, but only if the scattering cross section can be reliably quantified. This work achieves that for mountains covered with evergreen trees, and thus completes the task of mountain-area propagation modeling.

\section{Prediction of Multipath Delay Profiles}

The estimated value of $\gamma$ is used along with the Lambertian scattering model and the topo-map data directly in (4) and (5)

Fig. 4. Multipath delay profile—Cowichan Bay, near Victoria, BC, Canada.

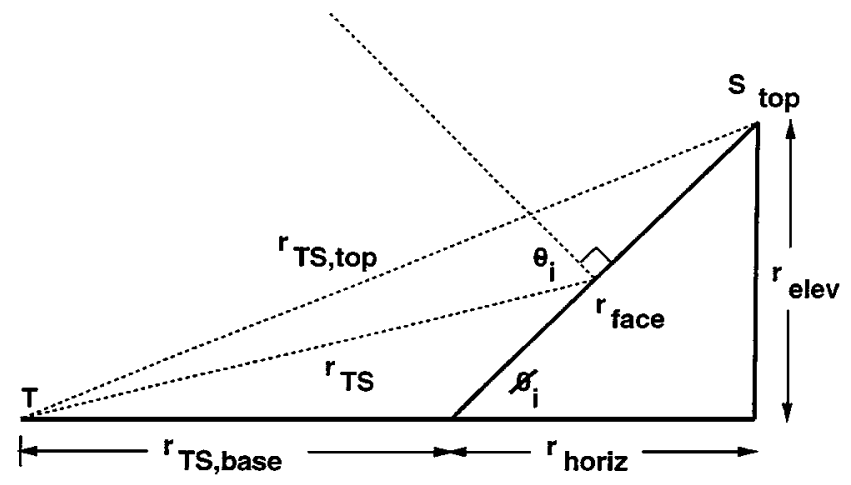

Fig. 5. Mountain slope geometry. 


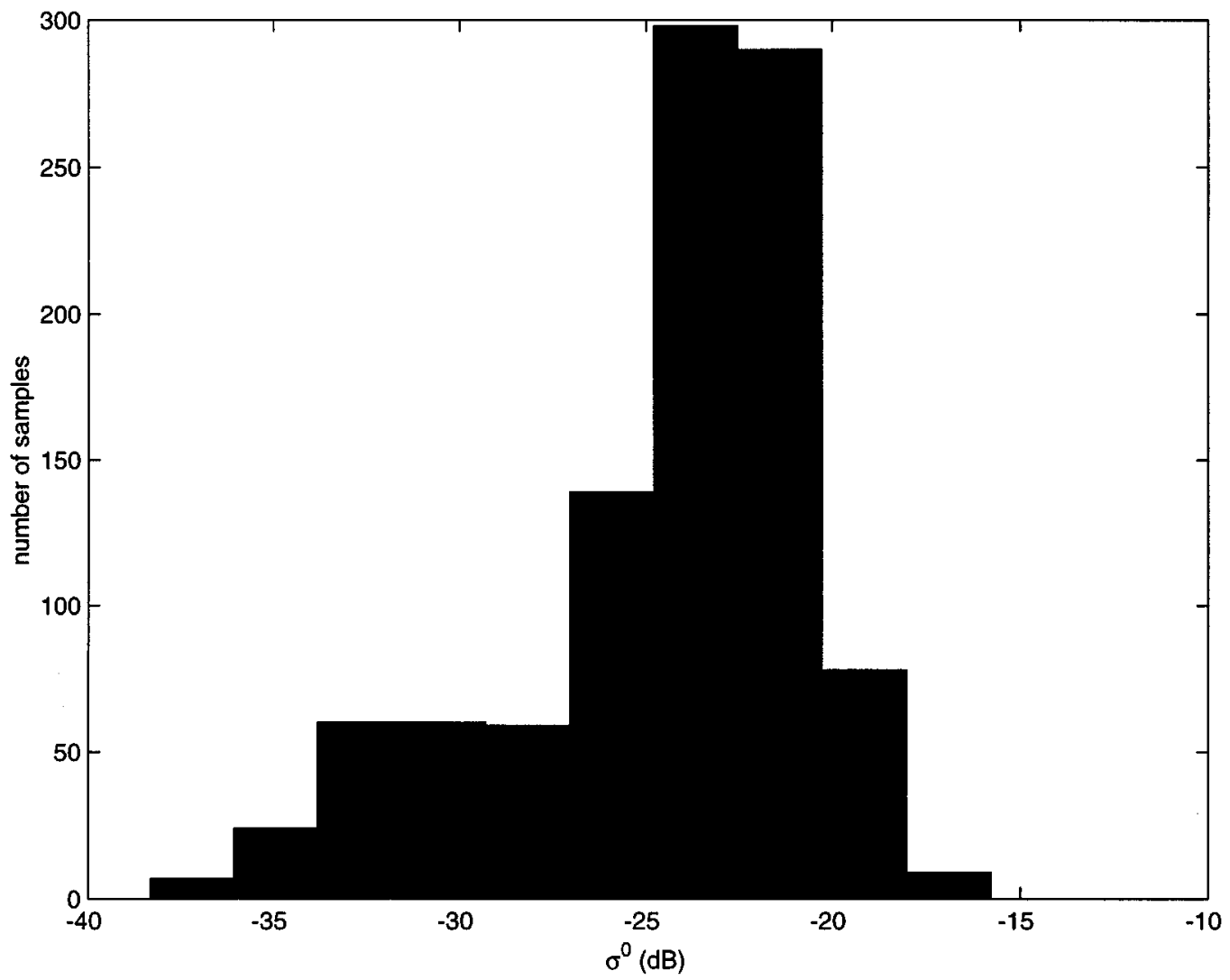

(a)

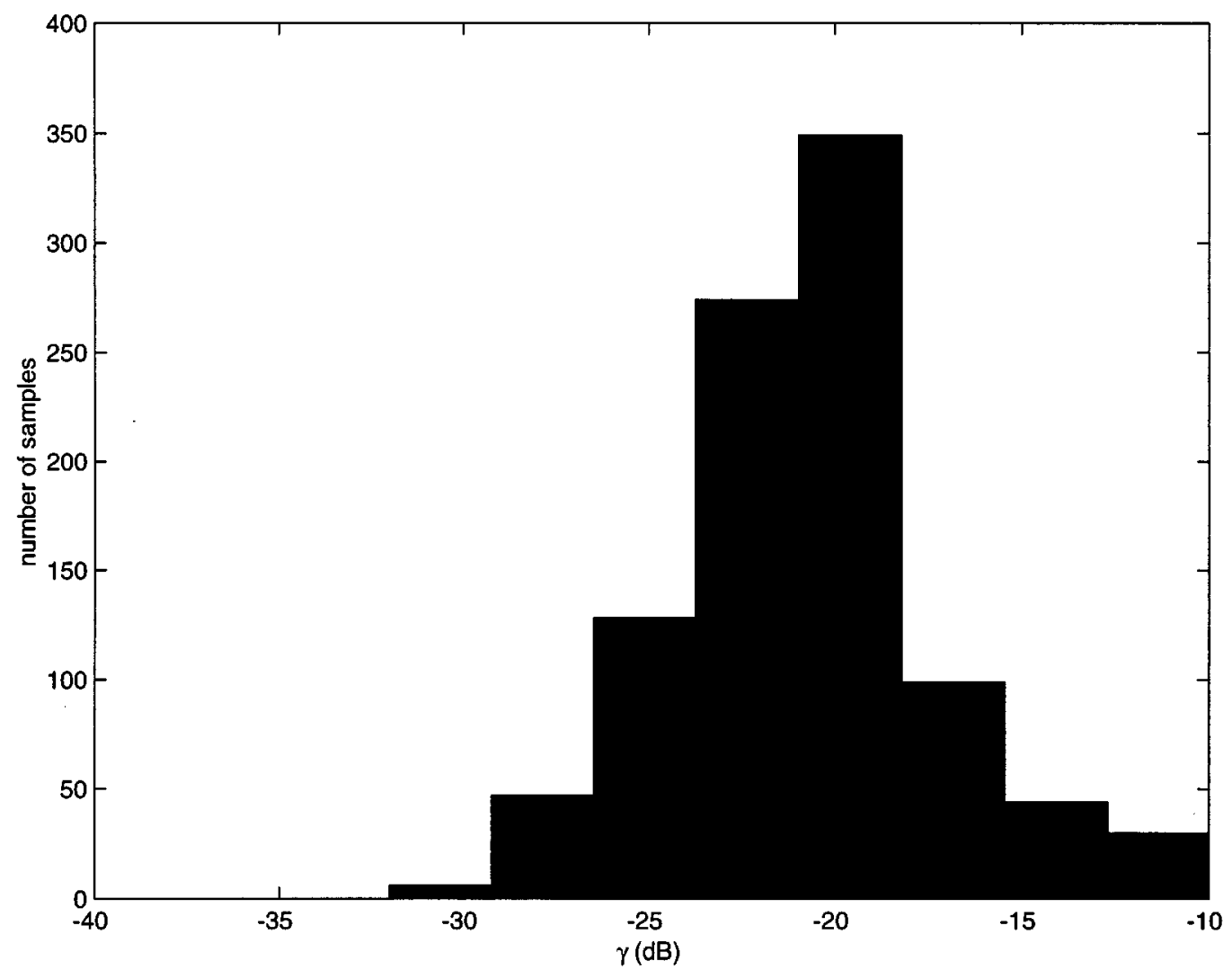

(b)

Fig. 6. (a) Histogram of normalized scattering cross section $\sigma_{0}$. (b) Histogram of reflectance $\gamma$. 
to predict the multipath delay profile. The MATLAB graphics package is used to render the topo-map surface using the Lambertian diffuse scattering model with specified locations for the "light source" (transmitter) and "camera" (receiver). Additional programming is needed to compute the multipath delay profile.

The "raw" multipath delay profile is a scatter plot of points representing amplitudes $L\left(t_{d}\right)$ versus delays as per (5), multiplied by $P_{T} G_{T} G_{R}$ to obtain $P_{r}(t)$ in units of power (dBm). These points, which are nonuniformly spaced along the delay axis, are combined with 4092 uniformly spaced points of complex Gaussian noise, scaled so that the total noise power $P_{N}$ corresponds to the expected value ( $-98 \mathrm{dBm}$ in this case). This aggregate of signal plus noise points is then filtered by adding all the powers within a time window around each point equal to the reciprocal bandwidth of the wireless system under consideration. The powers may be added, since there is no phase information in (5), and thus the obtained multipath delay profile represents a spatially averaged result, equivalent to averaging many delay profiles over several wavelengths. The maximum bandwidth should be no more than that equivalent to the space resolution of the terrain data base, e.g., $3 \mathrm{MHz}$ for a $100-\mathrm{m}$ data base. This filtering is equivalent to selecting only those elements $d A$ in (3) in a range cell for which $r_{T S}+r_{S R}$ is constant to within the distance resolution $c / B$.

The present radar equation model (4) assumes a line-of-sight path with free space propagation between source, scatterer and receiver. and needs to be refined to include diffraction or scattering losses along the path using one of the standard 2-D direct path propagation models. A sufficient refinement may be to include a term in (4) which represents any Fresnel zone losses for the $k$ th path if it grazes or comes near to an obstacle. Such a refinement could affect the shape of the multipath delay profile. The model (4) applies for omni as well as directional antennas.

Maps and corresponding multipath delay profiles (measured and predicted) are provided below for several sites selected from TableS II and III, along with detailed comments. For each map, the antenna pattern (approximated as a fan beam) is shown for $T$ and $R$, along with the elements of terrain area $d A$ where the beams overlap.

\section{A. Malahat 1 (Files motela-b), $S_{1}, R_{1}$, Fig. 1}

For file motela-b, the receiver $R_{1}$ has a clear and almost perpendicular view of a very uniform slope $S_{1}$ illuminated by $T$. The $T$ antenna has a $30^{\circ}$ beamwidth, the $R$ antenna is omnidirectional. The direct (LOS) path is not visible on the measured multipath delay profile. The predicted delay profile corresponds very well to the measured one in both amplitude and shape, except that there is a second peak in the predicted one. This second peak arises from elements of area $d A$ which are on slopes behind the main slope considered. The measured profile does not show these reflections, due to Fresnel zone losses.

\section{B. Malahat 2 (Files malpk), $S_{1}, R_{2}$, Fig. 2}

For files malpk, $R_{2}$ sees slope $S_{1}$ at a grazing angle less than $5^{\circ}$, where the calculation of $\gamma$ from the Lambertian model may not always be accurate [23]. Nonetheless, the predicted delay profile closely matches the measured one in both amplitude and shape. Similar results apply to files malpa for slope $S_{2}$.

\section{Cowichan Bay 1 (Files cbssa), $S_{2}, R_{1}$, Fig. 3 \\ D. Cowichan Bay 2 (Files cbb), $S_{2}, R_{2}$, Fig. 4}

Both $T$ and $R_{1}$ are off the map, $R_{1}$ with $10^{\circ}$ beamwidth is $200 \mathrm{~m}$ behind $T$ with $30^{\circ}$ beamwidth, hence the patches $d A$ where the two beams overlap appear in the center of the $T$ beam. $R_{1}$ sees two slopes $S_{2}$, a small one (Separation Point) and the large one behind (Mt. Sullivan, Bruce Peak), as can be clearly seen on the multipath delay profile. The predicted delay profile corresponds very well to the measured one in both amplitude and shape, However, the second (broad) peak is 3-6 dB lower in level than predicted, probably due to Fresnel zone losses from the first slope. Both slopes have similar measured $\sigma^{0}$ (Table III). As for Section VI-C above, $T$ is off the map. From $R_{2}$, only a few data files were obtained due to in-band interference from the radio towers on the mountain slope. Nonetheless, the predicted and measured delay profiles correspond quite well, except that the first measured peak is $10 \mathrm{~dB}$ stronger than predicted. The explanation is not clear, it may be due to a major reflector (e.g., water tower), or related to the interference.

Other sites in Table III not reported in detail.

Cowichan Bay: Looking at slope $S_{1}$ from both $R_{1}$ and $R_{2}$ (files cbaym-p and cbayd-i) the measured results for $\sigma^{0}$ are very close, again suggesting that the precise value of $\theta_{i}$ is not critical away from grazing or normal incidence [23], consistent with the Lambertian model.

Cowichan Lake : For files cowb-e, $R_{1}$ has a clear and almost perpendicular shot to the uniform slope $S_{1}$. The measured results for $\sigma^{0}$ are very consistent.

Vancouver: For $R_{1}$ (files brock) looking at slope $S_{1}$, the signal was weak and the measured $\sigma^{0}$ may not be reliable. For $R_{2}$ (files grouse, grous) looking at slope $S_{1}$, the receiver was so close to the mountain that the $30^{\circ}$ vertical beamwidth did not illuminate the entire slope. Tilting the array up $10^{\circ}$ from horizontal made $\mathrm{a} \simeq 6 \mathrm{~dB}$ difference in the measured value of $\sigma^{0}$.

\section{PREDiction of Complex IMPUlse Response}

Complex multipath delay profiles (impulse responses) are needed to predict wireless link performance. To evaluate the performance of a particular modulation/coding or equalization technique, the modulated signal is convolved with the positiondependent complex baseband impulse response $h\left(t, x_{T}, x_{R}\right)=$ $h(t, \tau)$ and then delivered to the receiver, where $x_{T}, x_{R}$ are the location coordinates of $T$ and $R$, and $\tau$ is the equivalent time dependence which is determined by the velocity of $T$ or $R$. Thus the predictions in the previous section must be modified to include phase information and generate a complex delay profile.

The phase cannot be predicted from the topographical map data with $\lambda \simeq 0.33 \mathrm{~m}$ and the map resolution of $100 \mathrm{~m}$. For a purely Lambertian surface $S_{k}$, where the surface roughness is of the order of $\lambda$ or greater, the scattering is diffuse, and the phase from each $S_{k}$ is uniformly distributed on $[0,2 \pi)$. Any specular 
TABLE III

MeAsurement Results $P_{T}=30 \mathrm{~dB}, G_{T}=14 \mathrm{~dB}, \theta_{T}=30^{\circ}$

files

Malahat

$\phi_{i}=33.7^{\circ} L^{\circ}\left(\phi_{i}\right)=2.5 \mathrm{~dB}$

motela1-50

motelb1-50

Malahat

$\phi_{i}=5^{\circ} L^{\circ}\left(\phi_{i}\right)=10.6 \mathrm{~dB}$

malpa121-140

malpk420-439

Cowichan Lake

$\phi_{i}=40^{\circ} L^{o}\left(\phi_{i}\right)=1.9 \mathrm{~dB}$

cowbl-100

cowe1-55

cowf1-50

cowcl-78

Cowichan Bay

$\phi_{i}=34^{\circ} L^{\circ}\left(\phi_{i}\right)=2.5 \mathrm{~dB}$

cbaym1-100

cbayn $1-80$

cbayp11-35

cbayd1-47

cbaye $1-50$

cbayg $11-70$

Cowichan Bay

$\phi_{i}=11^{\circ} L^{\circ}\left(\phi_{i}\right)=7.1 \mathrm{~dB}$

cbssb1-56

cbssa1-56

Cowichan Bay

$\phi_{i}=18^{\circ} L^{o}\left(\phi_{i}\right)=5.1 \mathrm{~dB}$

cbssa $1-56$

cbssb1-100

cbb39-50

cbb111-119 $t_{2}-t_{1} \quad G_{R}$

$(\mu \mathrm{sec}) \quad \mathrm{dB}$ $\theta_{R} \quad r_{T}$

$\begin{array}{lllllllll}4.75 & 2 & 360 & 1.9 & 2.3 & 0.855 & -21.3 & 1.3 & -18.8 \\ 4.75 & 2 & 360 & 1.9 & 2.3 & 0.855 & -19.8 & 1.2 & -17.3\end{array}$

$\begin{array}{lllllllll}15.25 & 20 & 10 & 2.5 & 3.2 & 1.5 & -35.3 & 1.5 & -24.8\end{array}$

$\begin{array}{lllllllll}10.75 & 20 & 10 & 2.5 & 3.2 & 0.9 & -32.7 & 2.0 & -22.1\end{array}$

Vancouver

$\phi_{i}=30^{\circ} L^{\circ}\left(\phi_{i}\right)=3.0 \mathrm{~dB} G_{T}=11 \mathrm{~dB}$ grouse $20-30$

grouse $76-90$

grous $36-50$

grous $71-90$

$\begin{array}{ll}18.7 & 20 \\ 18.7 & 20 \\ 18.7 & 20 \\ 18.7 & 20\end{array}$

$\begin{array}{lllllllll}4.7 & 2 & 360 & 2.194 & 2.319 & 1.0 & -22.6 & 0.8 & -20.7 \\ 4.7 & 2 & 360 & 2.194 & 2.319 & 1.0 & -24.3 & 1.8 & -22.4 \\ 4.7 & 2 & 360 & 2.194 & 2.319 & 1.0 & -23.7 & 1.6 & -21.8 \\ 4.7 & 2 & 360 & 2.194 & 2.319 & 1.0 & -20.2 & 1.1 & -18.3\end{array}$

$\begin{array}{lllllllll}6.35 & 2 & 360 & 2.921 & 1.978 & 1.0 & -24.5 & 2.6 & -22.0 \\ 6.5 & 2 & 360 & 2.921 & 1.978 & 1.0 & -22.3 & 1.7 & -19.8 \\ 6.5 & 11 & 15 & 2.921 & 1.978 & 0.504 & -22.5 & 1.5 & -20.0 \\ & & & & & & & & \\ 13.1 & 11 & 15 & 2.921 & 2.921 & 1.5 & -23.9 & 1.1 & -21.4 \\ 7.5 & 2 & 360 & 2.921 & 2.921 & 1.5 & -33.0 & 2.0 & -30.5 \\ 13.5 & 2 & 360 & 2.921 & 2.921 & 1.545 & -23.6 & 1.8 & -21.1\end{array}$

Vancouver

$\phi_{i}=30^{\circ} L^{\circ}\left(\phi_{i}\right)=3.0 \mathrm{~dB} G_{T}=14 \mathrm{~dB}$ brock 20-44

brock $112-116$

brock112-116

brock164-169

$\begin{array}{lllllllll}6.0 & 16 & 10 & 3.5 & 3.5 & 0.68 & -31.4 & 1.7 & -24.3\end{array}$

$\begin{array}{lllllllll}6.0 & 16 & 10 & 3.5 & 3.5 & 0.55 & -27.0 & 1.7 & -19.9\end{array}$

$\begin{array}{lllllllll}8.82 & 16 & 10 & 6.700 & 7.0 & 1.61 & -28.0 & 1.7 & -22.9 \\ 8.82 & 16 & 15 & 6.700 & 7.0 & 1.61 & -32.3 & 1.5 & -27.2 \\ & & & & & & & & \\ 20.0 & 20 & 10 & 8.5 & 6.5 & 3.5 & -17.5 & 1.3 & -12.4 \\ 15.0 & 20 & 10 & 8.5 & 6.5 & 2.5 & -24.5 & 2.6 & -19.6\end{array}$

components are reflected in directions away from $R .{ }^{12}$ This aggregate of signal points is then filtered by adding all the complex amplitudes within a distance window $c / B$ around each point. For a given point at given delay $t$, the envelope $|h(t)|^{2}$ shows Rayleigh-like fading when the position of $T$ or $R$ is changed (Fig. 7).

\footnotetext{
${ }^{1}$ Except in the case of a vertical wall, which is very rare in typical terrain.

${ }^{2}$ There may be some subpatches within $S_{k}$ : which yield a specular component where the phase is found from the path length from $T$ via $S_{k}$ to $R$. This would apply to man-made objects such as buildings or water towers.
}

For the purpose of convolving a modulated signal with the complex impulse response, $h(t)$ can be resampled at the sampling rate of the simulation to create a digital filter $h(n)$. The effect of motion of $R$ causing time variation in $h(t, \tau)$ is simulated by recomputing the tap weights $h(n)$ at each sample time $n$ according to the position of $R$ at time $n$. For example, an IS-136 simulation with symbol rate $24.3 \mathrm{~K}$ symbols/s, and four samples per symbol, moving $R$ in $0.45 \mathrm{~mm}$ intervals at each $n$ simulates a velocity of $100 \mathrm{~km} / \mathrm{h}$. One complete $2 \pi$ phase rotation occurs every $330 \mathrm{~mm}$ at $900 \mathrm{MHz}$, or every 733 samples. Noise is added 


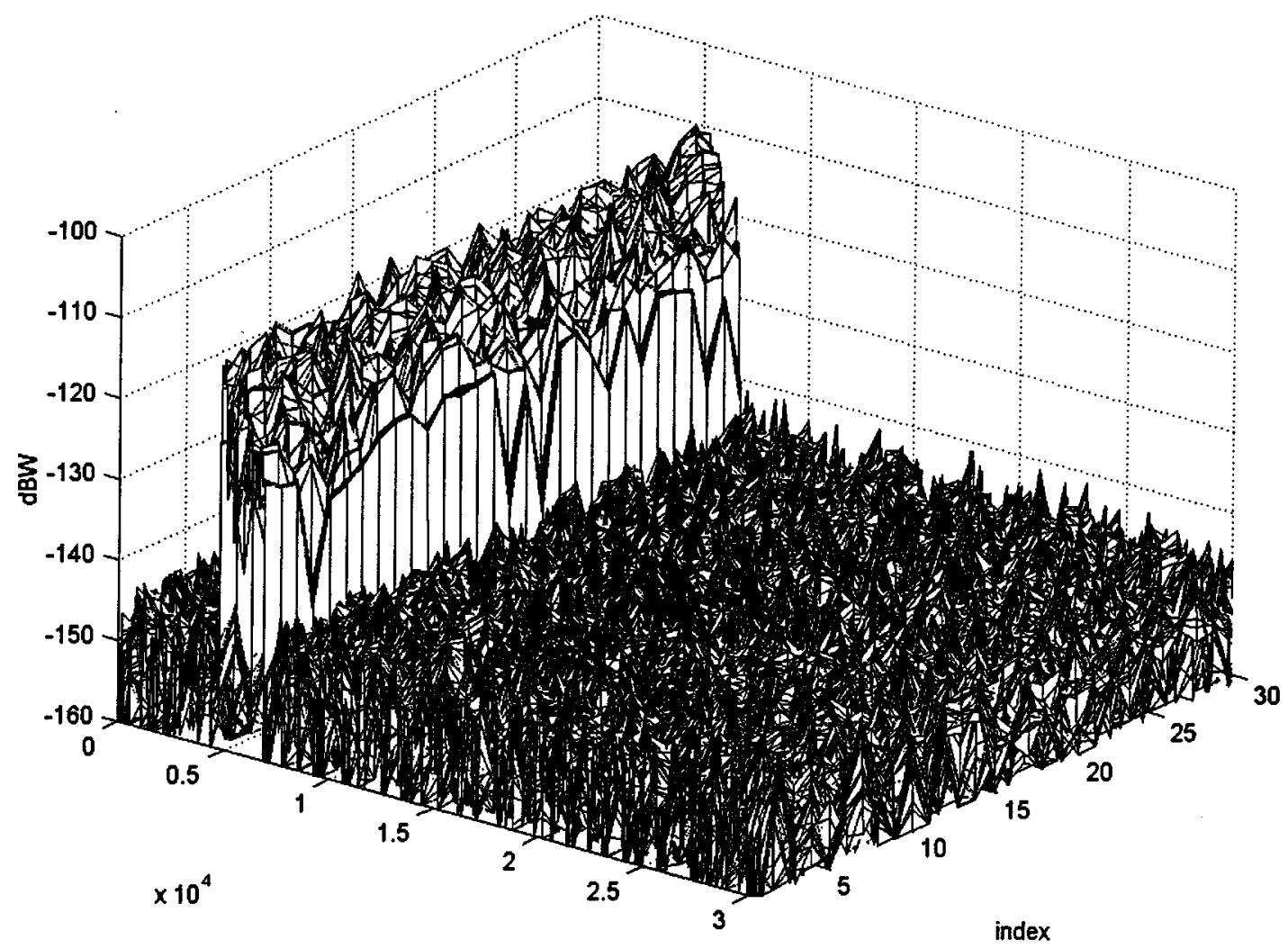

delay (meters, 3.3nsec/meter)

Fig. 7. Predicted multipath delay profiles, Malahat.

after the convolution and before the receiver filter to set the desired signal-to-noise ratio.

The first term in $h(t)$ representing the direct path between $T$ and $R$ is in practice not a $\delta$-function, and may be replaced by a cluster of $\delta$-functions with weights according to, e.g., the GSM typical urban (TU) or rural area (RA) profiles [27], and with total average power determined by any one of several standard propagation models. Thus the predicted impulse response is made up of two parts: a cluster corresponding to the direct path, plus another cluster arising from the terrain scattering.

\section{CONCLUSION}

Mountains act as large reflectors resulting in significant echoes at $900 \mathrm{MHz}$. The strongest long-delayed echoes are observed when both transmitter $T$ and receiver $R$ have a clear view of the mountains, and they have the most impact when the direct line-of-sight signal between them is blocked so that the echoes are comparable in strength to the direct signal.

A model of the impulse response was presented using the radar equation with normalized radar cross section $\sigma^{0}=\gamma \cos \theta_{i}$, where $\gamma$ is a property of the mountain slope. For the mountains near Victoria and Vancouver, Canada (wooded slopes not near vertical or horizontal), $\gamma$ was found to be remarkably consistent in the range $-21.1 \pm 2.9 \mathrm{~dB}$. This radar equation model was then used to predict the multipath delay profile in mountainous terrain from topographical data, and the predictions compared well with measurements. Thus these predictions can be repeated with confidence for a $T$ and
$R$ in any location on the map, and in turn, can be convolved with a simulated data stream to predict error rate, outage or other aspects of wireless system performance.

\section{APPENDIX \\ ESTIMATION OF $\rho_{m}$ FROM MEASURED IMPULSE RESPONSE SAMPLES}

The measured power in a cluster is obtained by processing the impulse response samples as follows: The total received power of an impulse response sample is given by the sum of the powers $\left(I^{2}+Q^{2}\right)$ at each delay (sampled at $25 \mathrm{~ns}$ intervals) in the range $0-102.3 \mu$ s (4092 samples per impulse response, four samples per PRBS chip). We define the signal-to-noise ratio

$$
\rho_{m}=\frac{\left(P_{S}+P_{N}\right)-P_{N}}{P_{N}}
$$

where the total received power $P_{S}+P_{N}$ for a cluster in time window $t_{1} \leq t \leq t_{2}$ is

$$
P_{S}+P_{N}=\sum_{t=t_{1}}^{t_{2}} P_{R}(t)
$$

and the noise power is calculated using a portion of the impulse response $t_{3} \leq t \leq t_{4}$ where there is no signal from

$$
P_{N}=\frac{4092}{t_{4}-t_{3}} \sum_{t=t_{3}}^{t_{4}} P_{R}(t)
$$

where $t_{i}, t$ are measured in units of the 25 -ns sampling period. The received noise power in the 20-MHz bandwidth is known to be $-98 \mathrm{dBm}$, thus providing a calibration of all computed powers in $\mathrm{dBm}$. 


\section{ACKNOWLEDGMENT}

The author would like to thank C. Zhang, Z. Gao, N. Kwon, J. Horton, H. Driessen, and S. Pridie for assisting with the measurements, and S. Burfoot, K. Atwal, and N. Agarwal for their assistance with the equipment setup in Victoria. He also thanks T. Brook for early work and finding [21] and the many people who provided access to private property locations that met the experimental criteria. He would also like to thank R. J. C. Bultitude of the Canadian Communications Research Center for supporting the original equipment development in 1991, A. J. Rustako and R. J. Roman for their major support to get set up with components, equipment, etc. He would also like to thank V. Erceg and L. Wang for their helpful feedback during and after presentations of interim results, $\mathrm{M}$. V. Clark for significant help with MATLAB programming, and L. J. Greenstein for initiating the work, many valuable suggestions, critical readings of this manuscript, and some text.

\section{REFERENCES}

[1] T. S. Rappaport, "Characterization of UHF multipath channels in factory buildings," IEEE Trans. Antennas Propagat., vol. 37, pp. 1058-1069, Aug. 1989.

[2] A. Turkmani et al., "Measurement and modeling of wideband mobile radio channels at $900 \mathrm{MHz}$," in Proc. Inst. Elect. Eng. I, vol. 138, Oct. 1991, pp. 447-457.

[3] J. P. De Weck, P. Merki, and R. W. Lorenz, "Power delay profiles measured in mountainous terrain," in Proc. IEEE Veh.. Tech. Conf., 1988, pp. $105-112$

[4] J. Shapira, "TERSCAT-A model for prediction of terrain scattered interference in microwave communication," in Record of the 16th Conf. Electrical and Electronics Engineers in Israel, Tel-Aviv, Mar. 1989, 2.2.4.

[5] J. Shapira, "Interference from mobile satellite systems through terrain scattering," Int. J. Wireless Inform. Networks, vol. 3, no. 3, pp. 173-179, 1996.

[6] W. C. Y. Lee and W. C. Y. Lee, Mobile Cellular Telecommunications Systems. New York: McGraw-Hill, 1989

[7] L. J. Greenstein, V. Erceg, Y. S. Yeh, and M. V. Clark, "A new path gain/delay spread popagation model for digital cellular channels," IEEE Trans. Veh. Technol., vol. 46, pp. 477-485, May 1997.

[8] P. Beckman and A. Spizzichino, The Scattering of Electromagnetic Waves from Rough Surfaces. New York: MacMillan, 1963.

[9] J. D. Parsons et al., "Sounding techniques for wideband mobile radio channels: A review," Proc. Inst. Elect. Eng. I, vol. 138, no. 5, pp. 437-446, Oct. 1991.

[10] W. E. Thomson and P. F. Carvalho, "VHF and UHF links using mountains as reflectors," IEEE Trans. Commun., vol. 26, pp. 391-400, Mar. 1978.

[11] P. F. Driessen, "Measured propagation characteristics of $900 \mathrm{MHz}$ mobile radio channels in mountainous terrain," in IEEE Vehicular Technology Conf., 1990, pp. 603-609.
[12] - "Multipath delay characteristics on mountainous terrain-Comparison of theoretical predictions with measurement results," in IEEE Vehicular Technology Conf., 1991, pp. 606-609.

[13] — "Multipath delay characteristics in mountainous terrain at 900 MHz," in IEEE Vehicular Technology Conf., Denver, CO, May 1992, pp. $520-523$.

[14] U. Liebenow and P. Kuhlman, "Theoretical investigations and wideband measurements on wave propagation in hilly terrain," in IEEE Vehicular Technology Conf., 1994, pp. 1803-1807.

[15] — - "A three-dimensional wave propagation model for macrocellular mobile communication networks in comparison with measurements," in IEEE Vehicular Technology Conf., 1996, pp. 1623-1627.

[16] P. Eggers et al., "Assessment of GSM-link quality dependence on radio dispersion in rural environments," in IEEE Vehicular Technology Conf., 1992, pp. 532-535.

[17] T. Maeyama, F. Ikegami, and Y. Kitano, "Analysis of mountain-reflected signal strength in digital mobile radio communications," IEICE Trans. Commun., vol. E76-B, pp. 98-102, Feb. 1993.

[18] A. Lie and P. K. Remvik, "Performance efficient method for scattering reflection prediction in mobile cellular networks," in IEEE Vehicular Technology Conf., 1997, pp. 1103-1107.

[19] R. L. Kirlin and P. F. Driessen, "Relationship between measured 900 $\mathrm{MHz}$ complex impulse responses and topographical map data," in IEEE Vehicular Technology Conf., 1994.

[20] T. Brook, P. F. Driessen, and R. L. Kirlin, "Propagation measurements using synthetic aperture radar techniques," in IEEE Vehicular Technology Conf., 1996.

[21] M. Oren and S. K. Nayar, "Generalization of the Lambertian model and implication for machine vision," Int. J. Comput. Vis., Feb. 1994.

[22] F. T. Ulaby and M. C. Dobson, Handbook of Radar Scattering Statistics for Terrain. Norwood, MA: Artech House, 1988

[23] M. W. Long, Radar Reflectivity of Land and Sea. Norwood, MA: Artech House, 1989

[24] A. K. Fung, Microwave Scattering and Emission Models and their Applications. Norwood, MA: Artech House, 1994.

[25] M2 Antenna Systems [Online] Available: http://www.m2inc.com/

[26] T. Takeuchi, S. Yoshida, and F. Ikegami, "Influence of mountain-reflected multipath delay on error performance in digital mobile radio," in Proc. URSI Int. Symp. Signals, Syst., Elec., 1989, pp. 369-372.

[27] R. Steele, Mobile Radio Communications. London, U.K.: Pentech, 1992, p. 728

Peter F. Driessen (S'76-M'79-SM'98) received the Ph.D. degree in electrical engineering from the University of British Columbia, Canada, in 1981.

From 1981 to 1985, Dr. Driessen was with several companies in Vancouver, Canada, working on projects for wireless data transmission and a custom VLSI modem chip. Since 1986, he has been with the University of Victoria, Victoria, BC, Canada, where he is currently an Associate Professor in the Department of Electrical and Computer Engineering. He was on sabbatical leave at AT\&T Laboratories, Holmdel, NJ, during the academic year 1992-1993, and at AT\&T Laboratories Research, Red Bank, NJ, during the academic year 1999-2000. From 1994 to 1999, he was an Editor for IEEE PERSONAL COMMUNICATIONS MAGAZINE and is currently an Editor for the IEEE JOURNAL ON SELECTED AREAS IN COMMUNICATIONS, Wireless Communications Series. His research interests are in the areas of wireless communications systems and redio propagation. 